\title{
NLO impact factor for diffractive dijet production in the shockwave formalism
}

\section{R. Boussarie*}

Institute of Nuclear Physics, Polish Academy of Sciences

Radzikowskiego 152, PL-31-342 Kraków, Poland

E-mail: Renaud.Boussariedifj.edu.pl

\section{A. V. Grabovsky}

Novosibirsk State University, 2 Pirogova street, Novosibirsk, Russia

Theory division, Budker Institute of Nuclear Physics, 11 Lavrenteva avenue, Novosibirsk, Russia

E-mail: a.v.grabovsky@inp.nsk.su

\section{Szymanowski}

National Centre for Nuclear Research (NCBJ), Hoża 69, 00-681 Warsaw, Poland

E-mail: Lech.Szymanowski@ncbj.gov.pl

\section{S. Wallon}

Laboratoire de Physique Théorique (UMR 8627), CNRS, Univ. Paris-Sud, Université Paris-Saclay, 91405 Orsay Cedex, France

UPMC, Université Paris 06, Faculté de Physique, 4 place Jussieu, 75252 Paris, France

E-mail: walloneth.u-psud.fr

We present the main steps of the computation of the impact factor for the exclusive diffractive photo- or electro- production of a forward dijet with NLO accuracy. In particular, we detail the cancellation mechanisms for all the divergences which appear in the intermediate results.

XXIV International Workshop on Deep-Inelastic Scattering and Related Subjects, 11-15 April, 2016, DESY Hamburg, Germany

\footnotetext{
* Speaker.
} 


\section{Introduction}

In this contribution, we report on our computation of the one loop $\gamma^{(*)} \rightarrow 2$ jets impact factor. This result is a first step toward a complete next-to-leading-order (NLO) description of many inclusive or exclusive diffractive processes, either in the linear BFKL [1, 2, 3, 4] or the non-linear color glass condensate (CGC) approaches $[5,6,7,8,9,10,11,12,13]$.

Two main approaches exist to theoretically describe diffraction: either through a resolved Pomeron contribution, see Fig. 1 (left), or using a direct Pomeron contribution involving the coupling of a Pomeron with the diffractive state, see Fig. 1 (right). Our results are based on the second one.
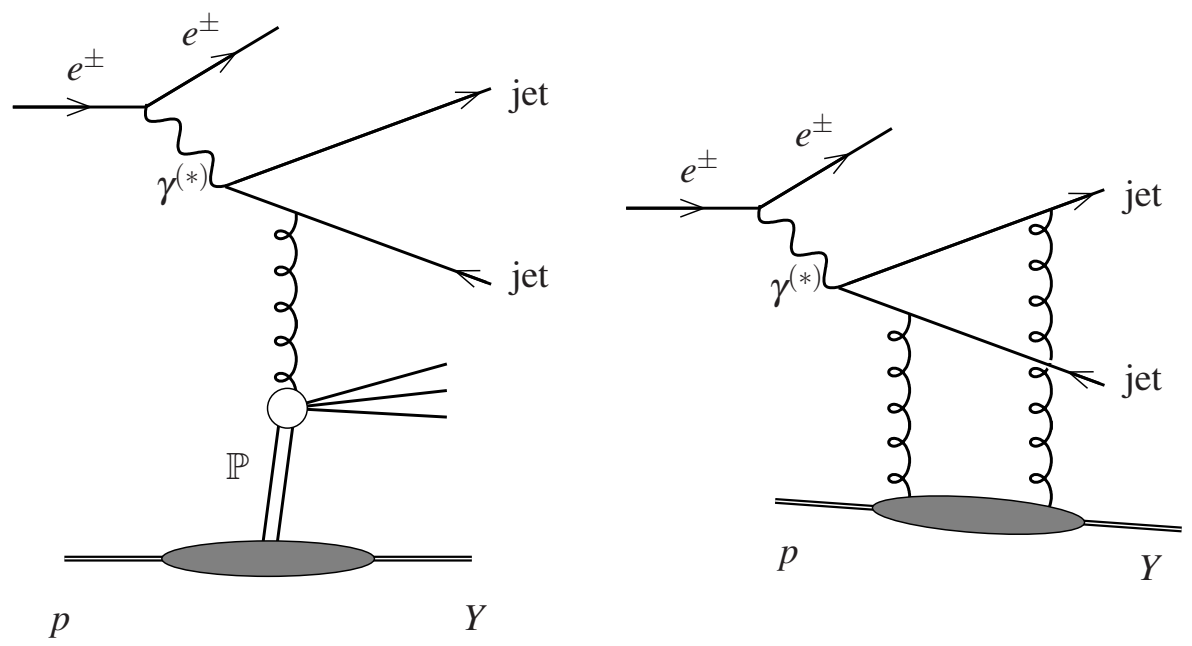

Figure 1: Resolved (left panel) and direct (right panel) Pomeron contributions to dijet production.

We show in particular that our result for the real contributions to the leading order (LO) $\gamma^{(*)} \rightarrow$ $q \bar{q} g$ impact factor and to the next-to-leading order (NLO) $\gamma^{(*)} \rightarrow q \bar{q}$ impact factor allows one to extract the finite part of the NLO impact factor for diffractive dijet production.

\section{The shockwave formalism in a nutshell}

Our calculation relies on Balitsky's QCD shockwave formalism [14, 15, 16, 17]. We introduce two lightcone vectors $n_{1}$ and $n_{2}$

$$
n_{1} \equiv(1,0,0,1), \quad n_{2} \equiv \frac{1}{2}(1,0,0,-1), \quad n_{1}^{+}=n_{2}^{-}=n_{1} \cdot n_{2}=1,
$$

and the Wilson lines as

$$
U_{i}^{\eta}=U_{\vec{z}_{i}}^{\eta}=T \exp \left[i g \int_{-\infty}^{+\infty} b_{\eta}^{-}\left(z_{i}^{+}, \vec{z}_{i}\right) d z_{i}^{+}\right] .
$$

The operator $b_{\eta}^{-}$is the external shockwave field built from slow gluons whose momenta are limited by the longitudinal cutoff $e^{\eta} p_{\gamma}^{+}$, where $\eta$ is an arbitrary negative parameter :

$$
b_{\eta}^{-}=\int \frac{d^{4} p}{(2 \pi)^{4}} e^{-i p \cdot z} b^{-}(p) \theta\left(e^{\eta}-\frac{\left|p^{+}\right|}{p_{\gamma}^{+}}\right)
$$




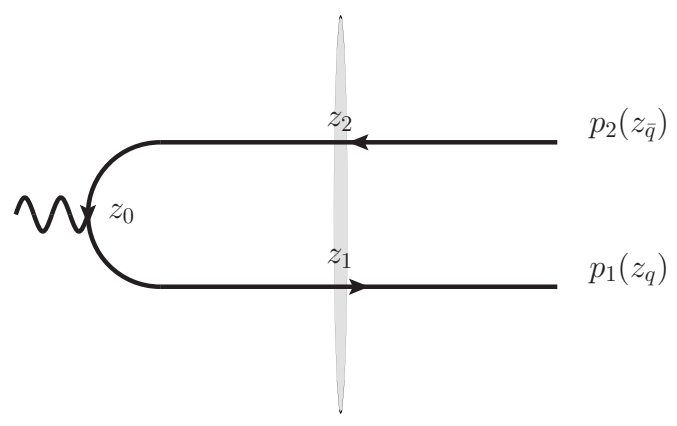

Figure 2: Leading order diagram for the impact factor for dijet production

where $p_{\gamma}$ is the momentum of the photon, which has a large component in the + direction.

We use the lightcone gauge $\mathscr{A} \cdot n_{2}=0$, with $\mathscr{A}$ being the sum of the external field $b_{\eta}$ and the quantum field $A_{\eta}$

$$
\mathscr{A}^{\mu}=A_{\eta}^{\mu}+b_{\eta}^{\mu}, \quad b_{\eta}^{\mu}(z)=b_{\eta}^{-}\left(z^{+}, \vec{z}\right) n_{2}^{\mu}=\delta\left(z^{+}\right) B_{\eta}(\vec{z}) n_{2}^{\mu},
$$

where $B_{\eta}(\vec{z})$ is a profile function and the form for $b_{\eta}$ is valid in the small $x$ limit considered here. From the Wilson lines, we define the dipole operator and its Fourier transforms as follows:

$$
\begin{aligned}
\mathbf{U}_{i j}^{\eta} & \equiv 1-\frac{1}{N_{c}} \operatorname{Tr}\left(U_{i}^{\eta} U_{j}^{\eta \dagger}\right), \\
\tilde{\mathbf{U}}_{i j}^{\eta} & \equiv \int d^{d} \vec{z}_{i} d^{d} \vec{z}_{j} e^{-i\left(\vec{p}_{i} \cdot \vec{z}_{i}\right)-i\left(\vec{p}_{j} \cdot \vec{z}_{j}\right)} \mathbf{U}_{i j}^{\eta}, \\
\widetilde{\mathbf{U}_{i k}^{\eta} \mathbf{U}_{k j}^{\eta}} & \equiv \int d^{d} \vec{z}_{i} d^{d} \vec{z}_{j} d^{d} \vec{z}_{k} e^{-i\left(\vec{p}_{i} \cdot \vec{z}_{i}\right)-i\left(\vec{p}_{j} \cdot \vec{z}_{j}\right)-i\left(\vec{p}_{k} \cdot \vec{z}_{k}\right)} \mathbf{U}_{i k}^{\eta} \mathbf{U}_{k j}^{\eta} .
\end{aligned}
$$

When computing a physical amplitude, one should act with these operators on the incoming and outgoing states of the target. For example in the case of a diffractive $\gamma^{(*)}\left(p_{\gamma}\right) P\left(p_{0}\right) \rightarrow X\left(p_{X}\right) P^{\prime}\left(p_{0}^{\prime}\right)$ process, the following matrix elements will be involved:

$$
\mathbf{W}^{\eta} \rightarrow\left\langle P^{\prime}\left(p_{0}^{\prime}\right)\left|T\left(\mathbf{W}^{\eta}\right)\right| P\left(p_{0}\right)\right\rangle
$$

where $\mathbf{W}^{\eta}$ is an operator built from the Wilson lines. In our case, there are two possibilities for $\mathbf{W}^{\eta}$ : either a dipole operator $\mathbf{W}^{\eta}=\mathbf{U}_{i j}^{\eta}$, or a double-dipole operator $\mathbf{W}^{\eta}=\mathbf{U}_{i k}^{\eta} \mathbf{U}_{k j}^{\eta}$. Note that in the t'Hooft limit $N_{c}^{-2} \rightarrow 0$ or in the mean field approximation, the matrix elements for the double dipole operators can be written as the product of the matrix elements for two dipole operators. From now on we will write $\mathbf{W}$ rather than $\mathbf{W}^{\eta}$ for readability.

\section{Impact factor for the $\gamma^{(*)} \rightarrow q \bar{q}$ transition}

At leading order, the diagram contributing to the impact factor for the $\gamma^{*} \rightarrow q \bar{q}$ transition is shown in Fig. 2. After the projection on the color singlet state and the subtraction of the contribution without interaction with the external field, the contribution of this diagram can be written in the momentum space as the following convolution of Wilson line operators with the impact factor:

$$
M_{L O}^{q \bar{q}}=\varepsilon_{\alpha} \int d \vec{p}_{1} d \vec{p}_{2} \delta\left(\vec{p}_{q 1}+\vec{p}_{\bar{q} 2}\right) \delta\left(p_{q}^{+}+p_{\bar{q}}^{+}-p_{\gamma}^{+}\right) \Phi_{0}^{\alpha}\left(\vec{p}_{1}, \vec{p}_{2}\right) \tilde{\mathbf{U}}_{12},
$$


where we denoted $p_{i j} \equiv p_{i}-p_{j}$, and where $p_{q}$ (resp. $p_{\bar{q}}$ ) is the momentum of the outgoing quark (resp. antiquark). $\Phi_{0}$ is directly obtained by computing the diagram in Fig. 2.
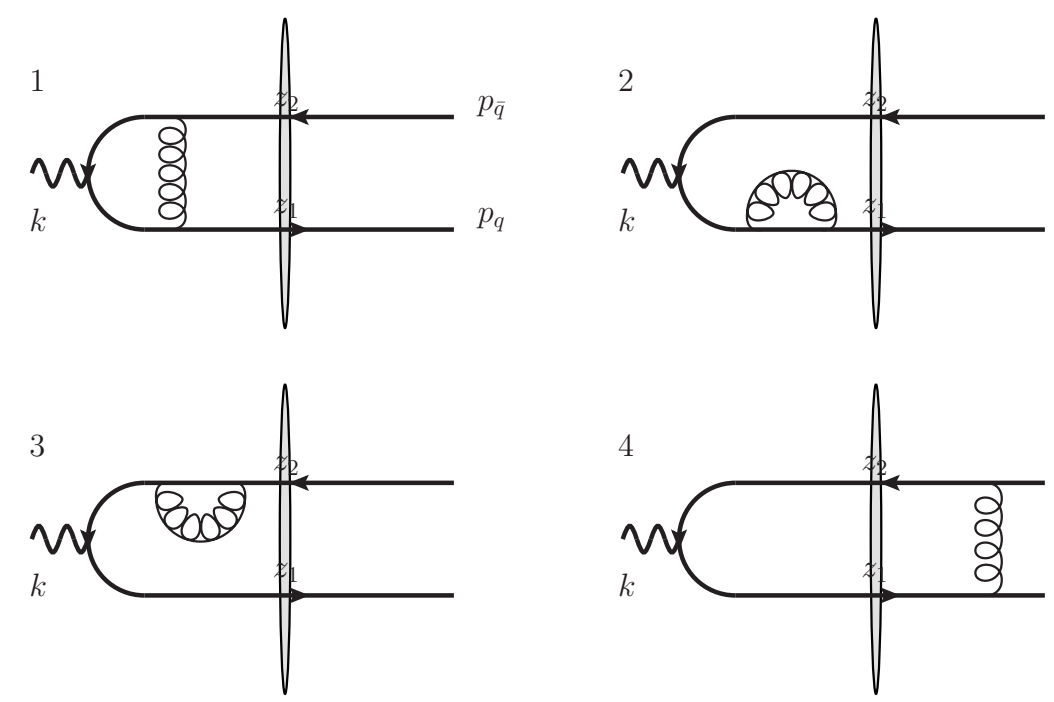

Figure 3: Diagrams contributing to the virtual corrections in which the radiated gluon does not cross the shockwave.

The virtual corrections to the $\gamma^{(*)} \rightarrow q \bar{q}$ transition involve two kinds of contributions. The diagrams contributing to virtual corrections in which the radiated gluon does not cross the shockwave field are shown in Fig. 3, and the diagrams in which the radiated gluon crosses the shockwave field are illustrated in Fig. 4. The convolution is similar to the leading order result, but it involves more Wilson line operators:

$$
\begin{aligned}
& M_{N L O}^{q \bar{q}}=\varepsilon_{\alpha} \int d^{d} \vec{p}_{1} d^{d} \vec{p}_{2} d^{d} \vec{p}_{3} \delta\left(\vec{p}_{q 1}+\vec{p}_{\bar{q} 2}-\vec{p}_{3}\right) \delta\left(p_{q}^{+}+p_{\bar{q}}^{+}-p_{\gamma}^{+}\right) \\
\times & \left\{\left(\frac{N_{c}^{2}-1}{N_{c}}\right) \tilde{\mathbf{U}}_{12} \delta\left(\vec{p}_{3}\right)\left[\Phi_{V_{1}}^{\alpha}+\Phi_{V_{2}}^{\alpha}\right]+N_{c}\left(\widetilde{\mathbf{U}_{13} \mathbf{U}_{32}}+\tilde{\mathbf{U}}_{13}+\tilde{\mathbf{U}}_{32}-\tilde{\mathbf{U}}_{12}\right) \Phi_{V_{2}}^{\alpha}\right\}
\end{aligned}
$$

where $\Phi_{V_{1}}^{\alpha}=\Phi_{V_{1}}^{\alpha}\left(\vec{p}_{1}, \vec{p}_{2}\right)$ is obtained from the diagrams in Fig. 3 and $\Phi_{V_{2}}^{\alpha}=\Phi_{V_{2}}^{\alpha}\left(\vec{p}_{1}, \vec{p}_{2}, \vec{p}_{3}\right)$ is obtained from the diagrams in Fig. 4.

Several divergences appear in each of the terms in Eq. (4.1): $\Phi_{V_{1}}^{\alpha}$ contains soft, collinear, soft and collinear, and UV divergences, while $\Phi_{V_{2}}^{\alpha}$ contains a rapidity divergence. In the shockwave formalism and in lightcone gauge, it is impossible to use the usual dimensional regularization around dimension 4 due to the presence of the cutoff on $p^{+}$momenta: the 2 longitudinal directions must be isolated. Thus we use dimensional regularization $d=2+2 \varepsilon$ for the transverse components, and the cutoff prescription $p^{+}<e^{\eta} p_{\gamma}^{+}$which is natural in our formalism.

The rapidity divergence in $\Phi_{V_{2}}$ is canceled via the use of the B-JIMWLK evolution equation for the dipole operator: evolving the dipole operator in the leading order convolution (3.1) w.r.t. the longitudinal cutoff from the arbitrary $e^{\eta} p_{\gamma}^{+}$to a more physical divide $e^{\eta_{0}} p_{\gamma}^{+}$, which will serve as a factorization scale which separates the upper and lower impact factors, allows one to cancel the dependence on $\eta$ in $\Phi_{V_{2}}$ and get a finite expression for the double-dipole contribution to the NLO 
impact factor. In momentum space and in $d+2$ dimensions, the evolution equation is given by:

$$
\begin{aligned}
& \frac{\partial \tilde{\mathbf{U}}_{12}^{\eta}}{\partial \log \eta}=2 \alpha_{s} N_{c} \mu^{2-d} \int \frac{d^{d} \vec{k}_{1} d^{d} \vec{k}_{2} d^{d} \vec{k}_{3}}{(2 \pi)^{2 d}} \delta\left(\vec{k}_{1}+\vec{k}_{2}+\vec{k}_{3}-\vec{p}_{1}-\vec{p}_{2}\right)\left(\widetilde{\left.\mathbf{U}_{13}^{\eta} \mathbf{U}_{32}^{\eta}+\tilde{\mathbf{U}}_{13}^{\eta}+\tilde{\mathbf{U}}_{32}^{\eta}-\tilde{\mathbf{U}}_{12}^{\eta}\right)}\right. \\
& \times\left[2 \frac{\left(\vec{k}_{1}-\vec{p}_{1}\right) \cdot\left(\vec{k}_{2}-\vec{p}_{2}\right)}{\left(\vec{k}_{1}-\vec{p}_{1}\right)^{2}\left(\vec{k}_{2}-\vec{p}_{2}\right)^{2}}+\frac{\pi^{\frac{d}{2}} \Gamma\left(1-\frac{d}{2}\right) \Gamma^{2}\left(\frac{d}{2}\right)}{\Gamma(d-1)}\left(\frac{\delta\left(\vec{k}_{2}-\vec{p}_{2}\right)}{\left[\left(\vec{k}_{1}-\vec{p}_{1}\right)^{2}\right]^{1-\frac{d}{2}}}+\frac{\delta\left(\vec{k}_{1}-\vec{p}_{1}\right)}{\left[\left(\vec{k}_{2}-\vec{p}_{2}\right)^{2}\right]^{1-\frac{d}{2}}}\right)\right] .(3.3)
\end{aligned}
$$

The divergences in $\Phi_{V_{1}}$ must be canceled by combining such terms with the associated real corrections to form a physical cross section. The first step to compute such a cross section is to use a jet algorithm in order to cancel the soft and collinear divergence. By using the jet cone algorithm in the small cone limit, as used in [18], we proved that such a cancellation occurs.

The remaining divergence can be expressed by factorizing the leading order cross section:

$$
d \sigma_{V d i v}^{\text {jets }}=\left(N_{V}+N_{V}^{*}\right) d \sigma_{L O}^{\text {jets }},
$$

where $N_{V}$ is extracted from the divergent part of the virtual amplitude. This contribution must be combined with real corrections from the $\gamma^{(*)} \rightarrow q \bar{q} g$ impact factor.
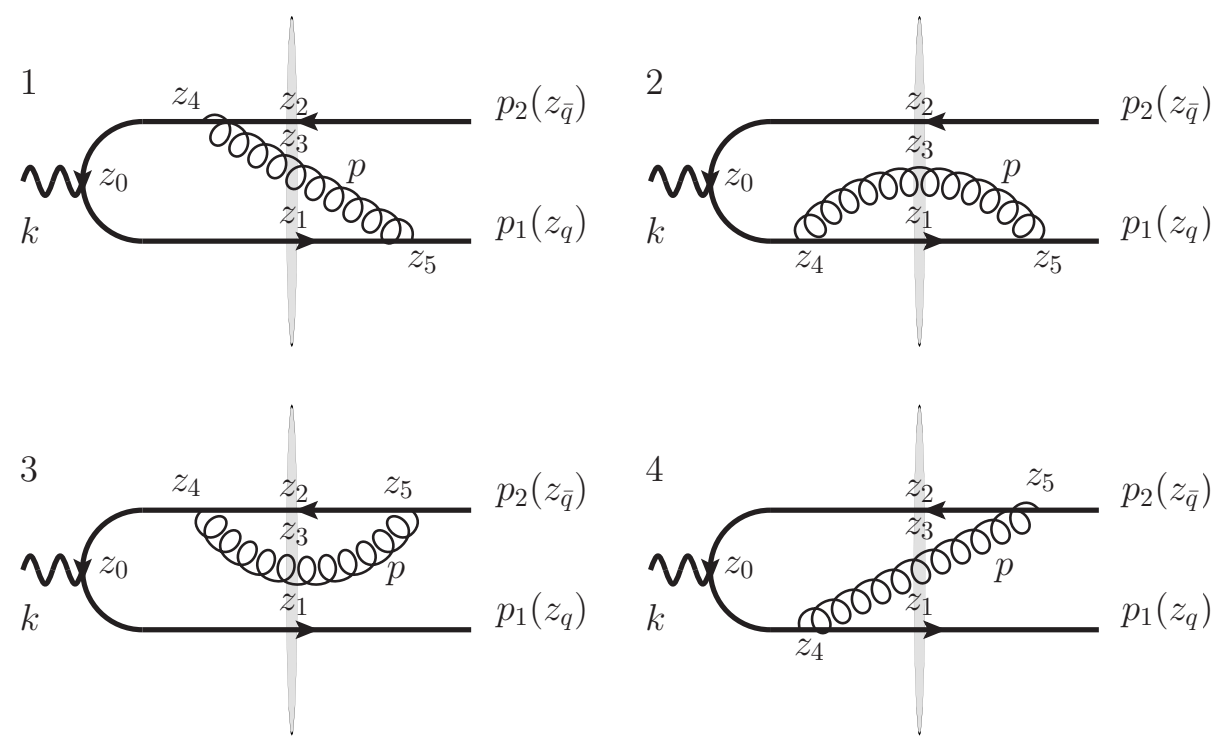

Figure 4: Diagrams contributing to the virtual corrections in which the radiated gluon interacts with the shockwave.

\section{Impact factor for the $\gamma^{(*)} \rightarrow q \bar{q} g$ transition}

The convolution for the $\gamma^{(*)} \rightarrow q \bar{q} g$ impact factor is very similar to the one for the NLO $\gamma^{(*)} \rightarrow$ $q \bar{q}$ impact factor: 



Figure 5: Diagrams contributing to real corrections to the impact factor for dijet production.

$$
\begin{aligned}
M^{q \bar{q} g}= & \varepsilon_{\alpha} \int d^{d} \vec{p}_{1} d^{d} \vec{p}_{2} d^{d} \vec{p}_{3} \delta\left(\vec{p}_{q 1}+\vec{p}_{\bar{q} 2}+\vec{p}_{g 3}\right) \delta\left(p_{q}^{+}+p_{\bar{q}}^{+}+p_{g}^{+}-p_{\gamma}^{+}\right) \\
\times & \left\{\left(\frac{N_{c}^{2}-1}{N_{c}}\right)\left[\Phi_{R_{1}}^{\alpha}+\Phi_{R_{2}}^{\alpha}\right] \tilde{\mathbf{U}}_{12} \delta\left(\vec{p}_{3}\right)\right. \\
& \left.+N_{c}\left(\widehat{\mathbf{U}_{13} \mathbf{U}_{32}}+\tilde{\mathbf{U}}_{13}+\tilde{\mathbf{U}}_{32}-\tilde{\mathbf{U}}_{12}\right) \Phi_{R_{2}}^{\alpha}\right\},
\end{aligned}
$$

where $\Phi_{R_{1}}=\Phi_{R_{1}}\left(\vec{p}_{1}, \vec{p}_{2}\right)$ and $\Phi_{R_{2}}=\Phi_{R_{2}}\left(\vec{p}_{1}, \vec{p}_{2}, \vec{p}_{3}\right)$ are obtained by computing respectively the first two diagrams and the last two diagrams in Fig. 5, as described in [19], [20] and [21]

When considering our exclusive cross section, the real contributions are those where the additional gluon is either collinear to the quark or to the antiquark, so that they form a single jet, or too soft to be detected i.e. with an energy which is lower than a typical energy resolution $E$. The contribution from the soft gluon to the dijet cross section can be written with a very simple form:

$$
d \sigma_{s o f t}^{q \bar{q} g}=\alpha_{s}\left(\frac{N_{c}^{2}-1}{2 N_{c}}\right) \int \frac{d p_{g}^{+}}{p_{g}^{+}} \frac{d^{d} \vec{p}_{g}}{(2 \pi)^{d}}\left|\frac{p_{q}}{\left(p_{q} \cdot p_{g}\right)}-\frac{p_{\bar{q}}}{\left(p_{\bar{q}} \cdot p_{g}\right)}\right|^{2} d \sigma_{L O}^{j e t s},
$$

where the integration is performed in the $p_{g}$-phase space region where $p_{g}^{+}+\frac{\vec{p}_{g}^{2}}{p_{g}^{+}}<2 E$.

The collinear contribution also has a simple form, in terms of the jet variables. For example when the gluon is collinear to the quark one gets:

$$
d \sigma^{(q g), \bar{q}}=\alpha_{s}\left(\frac{N_{c}^{2}-1}{2 N_{c}}\right) N_{J} d \sigma_{L O}^{j e t s}
$$

where $N_{J}$ is proportional to the «number of jets in the quark », a DGLAP-type emission kernel. As shown in [22], combining Eqs. (3.4), (4.2), (4.3) and the equivalent of Eq. (4.3) where the gluon is collinear to the antiquark, one finally obtains a finite cross section. 


\section{Conclusion}

Dijet production in DDIS at HERA was recently analyzed [23]. A precise comparison of dijet versus triple-jet production, which has not been performed yet at HERA [24], would be of much interest. Investigations of the azimuthal distribution of dijets in diffractive photoproduction performed by ZEUS [25] show signs of a possible need for a 2-gluon exchange model, which is part of the shockwave mechanism. Our calculation could be used for phenomenological studies of those experimental results. Complementary studies could be performed at LHC with UPC events.

\section{Acknowledgments}

A. V. Grabovsky acknowledges support of president scholarship 171.2015.2, RFBR grant 1302-01023, Dynasty foundation, Metchnikov grant and University Paris Sud. He is also grateful to LPT Orsay for hospitality while part of the presented work was being done. R. Boussarie thanks RFBR for financial support via grant 15-32-50219. This work was partially supported by the ANR PARTONS (ANR-12-MONU-0008-01), the COPIN-IN2P3 Agreement and the TheorieLHC France Initiative. L. Szymanowski was supported by grant No 2015/17/B/ST2/01838 of the National Science Center in Poland.

\section{References}

[1] V. S. Fadin, E. A. Kuraev, and L. N. Lipatov, On the Pomeranchuk Singularity in Asymptotically Free Theories, Phys. Lett. B60 (1975) 50-52.

[2] E. A. Kuraev, L. N. Lipatov, and V. S. Fadin, Multi - Reggeon Processes in the Yang-Mills Theory, Sov. Phys. JETP 44 (1976) 443-450.

[3] E. A. Kuraev, L. N. Lipatov, and V. S. Fadin, The Pomeranchuk Singularity in Nonabelian Gauge Theories, Sov. Phys. JETP 45 (1977) 199-204.

[4] I. I. Balitsky and L. N. Lipatov, The Pomeranchuk Singularity in Quantum Chromodynamics, Sov. J. Nucl. Phys. 28 (1978) 822-829.

[5] J. Jalilian-Marian, A. Kovner, A. Leonidov, and H. Weigert, The BFKL equation from the Wilson renormalization group, Nucl. Phys. B504 (1997) 415-431, [hep-ph / 9701284 ].

[6] J. Jalilian-Marian, A. Kovner, A. Leonidov, and H. Weigert, The Wilson renormalization group for low x physics: Towards the high density regime, Phys. Rev. D59 (1999) 014014, [hep-ph/ 9706377 ].

[7] J. Jalilian-Marian, A. Kovner, and H. Weigert, The Wilson renormalization group for low x physics: Gluon evolution at finite parton density, Phys. Rev. D59 (1999) 014015, [hep-ph / 9709432 ].

[8] J. Jalilian-Marian, A. Kovner, A. Leonidov, and H. Weigert, Unitarization of gluon distribution in the doubly logarithmic regime at high density, Phys. Rev. D59 (1999) 034007, [hep-ph/ 9807462 ].

[9] A. Kovner, J. G. Milhano, and H. Weigert, Relating different approaches to nonlinear QCD evolution at finite gluon density, Phys. Rev. D62 (2000) 114005, [hep-ph/ 0004014 ].

[10] H. Weigert, Unitarity at small Bjorken x, Nucl. Phys. A703 (2002) 823-860, [hep-ph / 0004044 ].

[11] E. Iancu, A. Leonidov, and L. D. McLerran, Nonlinear gluon evolution in the color glass condensate. I, Nucl. Phys. A692 (2001) 583-645, [hep-ph/ 0011241$].$ 
[12] E. Iancu, A. Leonidov, and L. D. McLerran, The renormalization group equation for the color glass condensate, Phys. Lett. B510 (2001) 133-144, [hep-ph/ 0102009 ].

[13] E. Ferreiro, E. Iancu, A. Leonidov, and L. McLerran, Nonlinear gluon evolution in the color glass condensate. II, Nucl. Phys. A703 (2002) 489-538, [hep-ph/ 0109115$].$

[14] I. Balitsky, Operator expansion for high-energy scattering, Nucl. Phys. B463 (1996) 99-160, [hep-ph/9509348].

[15] I. Balitsky, Factorization for high-energy scattering, Phys. Rev. Lett. 81 (1998) 2024-2027, [hep-ph/9807434].

[16] I. Balitsky, Factorization and high-energy effective action, Phys. Rev. D60 (1999) 014020, [hep-ph/9812311].

[17] I. Balitsky, Effective field theory for the small-x evolution, Phys. Lett. B518 (2001) 235-242, [hep-ph/0105334].

[18] D. Y. Ivanov, M. I. Kotsky, and A. Papa, The impact factor for the virtual photon to light vector meson transition, Eur. Phys. J. C38 (2004) 195-213, [hep-ph/ 0405297 ].

[19] G. Beuf, NLO corrections for the dipole factorization of DIS structure functions at low x, Phys. Rev. D85 (2012) 034039, [arXiv:1112.4501].

[20] R. Boussarie, A. Grabovsky, L. Szymanowski, and S. Wallon, Impact factor for high-energy two and three jets diffractive production, JHEP 1409 (2014) 026, [arXiv: 1405 . 7676].

[21] A. Ayala, M. Hentschinski, J. Jalilian-Marian, and M. E. Tejeda-Yeomans, Polarized 3 parton production in inclusive DIS at small x, Phys. Lett. B761 (2016) 229-233, [arXiv: 1604.0852 ].

[22] R. Boussarie, A. V. Grabovsky, L. Szymanowski, and S. Wallon, On the one loop $\gamma^{(*)} \rightarrow q \bar{q}$ impact factor and the exclusive diffractive cross sections for the production of two or three jets, arXiv: 1606.0041.

[23] H1 Collaboration Collaboration, F. Aaron et. al., Measurement of Dijet Production in Diffractive Deep-Inelastic Scattering with a Leading Proton at HERA, Eur. Phys. J. C72 (2012) 1970, [arXiv:1111.0584].

[24] H1 Collaboration Collaboration, C. Adloff et. al., Diffractive jet production in deep inelastic $e^{+} p$ collisions at HERA, Eur. Phys. J. C20 (2001) 29-49, [hep-ex/ 0012051$].$

[25] ZEUS Collaboration, M. Guzik, Exclusive dijet production in diffractive deep inelastic scattering at HERA, PoS DIS2014 (2014) 070. 